OPEN ACCESS

Edited by:

Eminy Hsiao-Yuan Lee, Institute of Biomedical Sciences, Academia Sinica, Taipei, Taiwan

Reviewed by:

Yi-Hsuan Lee,

National Yang-Ming University, Taiwan

Pei-Ning Wang,

National Yang-Ming University, Taiwan

*Correspondence:

Jiun-Jie Wang

jwang@mail.cgu.edu.tw

${ }^{\dagger}$ These authors have contributed equally to this work.

Received: 03 October 2016 Accepted: 14 December 2016

Published: 11 January 2017

Citation:

Lin S-H, Hsu W-C, Ng S-H, Cheng J-S, Khegai O, Huang C-C,

Chen Y-L, Chen Y-C and Wang J-J (2017) Increased Water Diffusion in the Parcellated Cortical Regions from the Patients with Amnestic Mild Cognitive Impairment and Alzheimer's Disease.

Front. Aging Neurosci. 8:325. doi: 10.3389/fnagi.2016.00325

\section{Increased Water Diffusion in the Parcellated Cortical Regions from the Patients with Amnestic Mild Cognitive Impairment and Alzheimer's Disease}

\author{
Sung-Han Lin ${ }^{1,2 t}$, Wen-Chuin $\mathrm{Hsu}^{3,4 t}$, Shu-Hang $\mathrm{Ng}^{1,5}$, Jur-Shan Cheng ${ }^{6}$, \\ Oleksandr Khegai ${ }^{1}$, Chin-Chang Huang ${ }^{7}$, Yao-Liang Chen ${ }^{5,8}$, Yi-Chun Chen ${ }^{3,4}$ and \\ Jiun-Jie Wang ${ }^{1,9,10,11,12 *}$
}

\begin{abstract}
${ }^{1}$ Department of Medical Imaging and Radiological Sciences, Chang Gung University, Taoyuan, Taiwan, ${ }^{2}$ Graduate Institute of Clinical Medical Sciences, Chang Gung University, Taoyuan, Taiwan, ${ }^{3}$ Department of Neurology, Chang Gung Memorial Hospital, Taoyuan, Taiwan, ${ }^{4}$ Dementia Center, Chang Gung Memorial Hospital, Taoyuan, Taiwan, ${ }^{5}$ Department of Medical Imaging and Intervention, Chang Gung Memorial Hospital, Linkou, Taiwan, ${ }^{6}$ Clinical Informatics and Medical Statistics Research Center, College of Medicine, Chang Gung University, Taoyuan, Taiwan, ${ }^{7}$ Department of Neurology, Chang Gung Memorial Hospital, Chang Gung University, College of Medicine, Taoyuan, Taiwan, ${ }^{8}$ Department of Diagnostic Radiology, Chang Gung Memorial Hospital, Keelung, Taiwan, ${ }^{9}$ Department of Medical Imaging and Radiological Sciences, Healthy Aging Research Center, College of Medicine, Chang Gung University, Taoyuan, Taiwan, ${ }^{10}$ Neuroscience Research Center, Chang Gung Memorial Hospital, Taoyuan, Taiwan, " Medical Imaging Research Center, Institute for Radiological Research, Chang Gung University/Chang Gung Memorial Hospital, Taoyuan, Taiwan, ${ }^{12}$ Center for Advanced Molecular Imaging and Translation, Chang Gung Memorial Hospital, Linkou, Taiwan
\end{abstract}

Background: The loss of cortical neuron environment integrity is the hallmark of neurodegeneration diseases such as Alzheimer's disease (AD) and amnestic mild cognitive impairment (aMCl). To reveal the microenvironment changes in cerebral cortex, the current study aimed to examine the changes of mean diffusivity (MD) in parcellated brain among $\mathrm{AD}, \mathrm{aMCl}$ patients and normal controls $(\mathrm{NC})$.

Methods : Diffusion tensor imaging data with the whole brain coverage were acquired from $28 \mathrm{AD}$ (aged 69.4 \pm 8.2 year old), $41 \mathrm{aMCl}$ patients (aged 68.2 \pm 6.4 year old) and $40 \mathrm{NC}$ subjects (aged $65.7 \pm 6.4$ year old). Subsequently, the MD values were parcellated according to the standard automatic anatomic labeling (AAL) template. Only the 90 regions located in the cerebral cortex were used in the final analysis. The mean values of MD from each brain region were extracted and compared among the participant groups. The integrity of the white matter tracts and gray matter atrophy was analyzed using the track-based spatial statistics and voxel-based morphometry approaches, respectively.

Results : Significant differences of MD were noticed both in aMCl and $\mathrm{AD}$ patients, in terms of the affected regions and the amount of increase. The hippocampus, parahippocampal gyrus and cingulum were the most significantly affected regions in $A D$ patients. From all the 90 cerebral cortex regions, significant increase of $M D$ in the $A D$ patients was found in 40 regions, compared to only one (fusiform gyrus on the right) in 
aMCl patients. In the disease affected regions, the $\mathrm{MD}$ from aMCl patients is in state between $\mathrm{NC}$ and $\mathrm{AD}$ patients.

Conclusions : Increased MD in the specific regions of the brain shows the feasibility of $\mathrm{MD}$ as an indicator of the early stage cortical degeneration in $\mathrm{aMCl}$ and $\mathrm{AD}$ patients.

Keywords: Alzheimer disease, mild cognitive impairment, cerebral cortex, diffusion MRI, parcellation

\section{INTRODUCTION}

Alzheimer's disease (AD), a progressive neurodegenerative disease, is the most common form of dementia (Alzheimer's, 2014). In 2014, nearly 44 million population worldwide were affected by Alzheimer's or related dementia and expected to almost triple in 2050 (Prince et al., 2014). However, only 1in-4 people with $\mathrm{AD}$ have been correctly diagnosed (Prince et al., 2014). Neuropathological studies showed that patients with $\mathrm{AD}$ are often accompanied with the deposition of amyloid and neurofibrillary tangle (NFT), which initiates from the temporal lobe and subsequently spreads out to the whole brain in the later stage (Braak and Braak, 1991). The accumulation of amyloid and NFT leads to neural death and brain shrinkage (Braak and Braak, 1991). Patients with AD suffer from the loss of brain functions, which typically starts with the impairment of the short-term memory in the early stage and extends to the long-term memory loss. The typical survival time from onset of $\mathrm{AD}$ to death varies from 4 to 8 years after the first diagnosis.

Mild cognitive impairment (MCI) refers to a specific group of patients who show deficits in the neuropsychological performance but do not satisfy the criteria of dementia (Petersen et al., 2001). Patients with amnestic type MCI (aMCI) have early memory complaints either with or without impairment in additional cognitive domains (Petersen et al., 1999). After a follow-up of 6 years approximately $80 \%$ of aMCI patients are diagnosed with AD (Petersen et al., 1999). It was noticed that this specific subgroup of patients will convert to $\mathrm{AD}$ at a rate 3 6 times higher when compared with the healthy subjects (Thompson et al., 2007). As a result, patients with aMCI are considered as a high-risk group of precursory AD (Prince et al., 2014). The understanding of the neuro-structural alterations in patients with aMCI, as related to the memory impairment, will potentially lead to a new insight of the underlying pathogenesis mechanism of $\mathrm{AD}$.

Magnetic resonance imaging (MRI), a noninvasive imaging tool and free from ionizing radiation, has been used to study the extent of neuro-structural degeneration in patients with $\mathrm{AD}(\mathrm{Du}$ et al., 2001; Thompson et al., 2007; Whitwell et al., 2008; Burton et al., 2009). The typical volumetric measurement in the brains of patients with $\mathrm{AD}$ reported atrophy in medial temporal lobe (MTL), hippocampus and the entorhinal cortex (Du et al., 2001; Burton et al., 2009). Similarly, atrophy in patients with aMCI also starts from hippocampus and entorhinal cortex (Whitwell et al., 2008). Post-mortem studies (Braak and Braak, 1991) showed that $\mathrm{AD}$ affected neurodegeneration sequence could initiate from medial temporal lobe and subsequently involve the whole brain. This similarity in the brain atrophy pattern further supports the hypothesis that aMCI could be a transition stage between the healthy status and AD (Ahmed et al., 2008). However, the brain atrophy by itself is not specific to the patients with $\mathrm{AD}$. The morphological changes can only be detected at an advanced stage when the volume loss is no longer recoverable. Therefore, an image probe that is capable of detecting the subtle changes in the brain at an earlier stage of the disease is highly desirable.

Diffusion tensor imaging (DTI) allows for the measurement of micro-scale displacement of water molecules using MRI in a non-invasive manner. It could measure the water diffusivity in the brain in vivo, which is a reflection of the brain tissue integrity (Basser and Pierpaoli, 1996). Mean diffusivity (MD) is the average of water diffusivity measured along all directions (Basser and Pierpaoli, 1996). In a free and isotropic environment, the measured MD is approximately $3 \times 10^{-3} \mathrm{~mm}^{2} / \mathrm{s}$ (Helenius et al., 2002). In the biological environment, water diffusion could be restricted and hindered. $\mathrm{MD}$ is approximately $0.89 \times 10^{-3}$ $\mathrm{mm}^{2} / \mathrm{s}$ in the cortical gray matter and $0.70 \times 10^{-3} \mathrm{~mm}^{2} / \mathrm{s}$ in white matter (Helenius et al., 2002).

Because the alteration in neuronal function might precede the structural atrophy, the current study proposed to investigate the value of changes in MD as an early sign of the neurodegeneration sequence in the natural course of Alzheimer Disease. Previous DTI studies on AD and/or aMCI mostly focused on either the changes in white matter tracts or the hypothesis-driven regions of interest such as hippocampus, entorhinal cortex and its neighborhood (Fellgiebel et al., 2004; Mielke et al., 2009). The most vulnerable white matter tracts involve uncinate fasciculus, superior longitudinal fasciculus, inferior longitudinal fasciculus and splenium of the corpus callosum (Liu et al., 2011; Wai et al., 2014), indicating a global influence of the brain from the aMCI and $\mathrm{AD}$. Significantly increased $\mathrm{MD}$ in pathologically related structures can be found in hippocampus, cingulum and corpus callosum (Fellgiebel et al., 2004). However, the white matter degeneration could be secondary to the gray matter damage (Zhang et al., 2009). Therefore, the investigation into the general involvement of MD in the cortex is of great interest.

The MD value is a reflection of the balance between the different cellular contributions. Because the water diffusivity from the extracellular space could be larger than the intracellular component, the increased $\mathrm{MD}$ is often related to an increased extracellular volume (Duong et al., 1998), as a result of the collapse of neural integrity (Schaefer et al., 2000). The increased $\mathrm{MD}$ has been reported in the patients with neurodegenerative diseases, for example, Parkinson's disease, and was attributed to the microstructural alterations such as neural death ( $\mathrm{Lu}$ et al., 2016). Because the neurodegeneration sequence often begin with the loss of neural integrity and result in the cell death in the end 
(Tripathi, 2012), the MD might showed increased sensitivity to the early sign of neurodegeneration.

In the present study, the hypothesis is that different extents of cortical damages between aMCI and AD can be detected by MD. Therefore, this study aimed to investigate the changes in $\mathrm{MD}$ as measured in cerebral cortex and assessed its potential as an early marker of neurodegeneration. Mean diffusivity from the parcellated cortical brain regions were measured. The differences between aMCI and $\mathrm{AD}$ in each region were examined. The correlation between the $\mathrm{MD}$ and cognitive performance was analyzed.

\section{METHODS}

The study was approved by the Institutional Review Board of Chang Gung Medical Foundation in Linkou, Taiwan, and complied with the Declaration of Helsinki. All participants gave written informed consent before participating the study.

\section{Participants}

The participants were divided into three age-range matched groups, which consists of 40 healthy normal controls (NC, Male/Female $=22 / 18$, aged $65.4 \pm 6.2$ year old), 41 patients with aMCI (Male/Female $=20 / 21$, aged $68.3 \pm 6.7$ year old) and 28 patients with $\mathrm{AD}$ (Male/Female $=11 / 17$, aged $68.5 \pm$ 7.9 year old). All subjects were screened by a medical history review, physical examination, and neuropsychological testing. The physical examination, and neuropsychological testing was performed within 2 weeks of the MR examinations. Four key cognitive domains were assessed: memory, executive function, language and visuospatial skills. The neuropsychological assessments performed in this study including the MiniMental State Examination (MMSE), clinical dementia rating (CDR); word sequence learning-recall, visual reproduction, logic memory II in the memory function evaluation; semantic association of verbal fluency, Wisconsin Card Sorting Test (WCST) completed categories, Wechsler Adult Intelligence Scale-III (WAIS-III) digit symbol-scaled, WAIS-III digit spanscaled in executive function evaluation; 3-D block construction Models in visuospatial evaluation, and object naming test in the language function evaluation. Subjects with depressive symptoms were excluded by using either the Hamilton Depression Rating Scale (NC) or the Cornell scale for depression in dementia (AD and aMCI). The general exclusion criteria for MRI examination also applied. The Apolopoprotein E status were also examined if available.

The diagnosis of $\mathrm{AD}$ was made by using NINCDS-ADRDA criteria (McKhann et al., 1984). The MCI patients were determined by the criteria of Petersens et al. (Petersen, 2004). An aMCI patient was defined as patient with MCI and memory impairment. The healthy controls were identified as cognitively normal, judged by clinical assessment and neuropsychological testing. The demographic data are summarized in Table 1.

\section{Image Acquisition}

Images were acquired using a $3 \mathrm{~T}$ MR scanner (MAGNETOM Trio a TIM system, Siemens, Erlangen, Germany). T2
TABLE 1 | General characteristics of the study participants and neuropsychological evaluation scores.

\begin{tabular}{|c|c|c|c|}
\hline & Control $(n=40)$ & $\mathrm{aMCl}(n=41)$ & $\mathrm{AD}(n=28)$ \\
\hline Mean age & $65.4(6.2)$ & $68.3(6.7)$ & $68.3(7.7)$ \\
\hline Gender (Male/Female) & $22 / 18$ & $20 / 21$ & $11 / 17$ \\
\hline Education & $10.4(4.3)^{\star}$ & $6.8(5.1)$ & $6.1(5.2)^{\#}$ \\
\hline Hypertension (w/o) & $8 / 40$ & $24 / 41$ & $11 / 28$ \\
\hline \multicolumn{4}{|l|}{ APOLIPOPROTEIN E STATUS } \\
\hline$\varepsilon 4$ carriers & 0 & 9 & 4 \\
\hline$\varepsilon 4$ noncarriers & 22 & 14 & 12 \\
\hline Unknown & 18 & 18 & 12 \\
\hline \multicolumn{4}{|l|}{ MEAN GLOBAL SCORE } \\
\hline MMSE (Maximum 30) & $28.6(1.1)^{\star}$ & $23.7(4.9)^{\#}$ & $14.6(6.1)^{\# \$}$ \\
\hline Clinical dementia rating (CDR) & $0.0(0.0)^{*}$ & $0.5(0.0)^{\#}$ & $1.0(0.6)^{\# \$}$ \\
\hline \multicolumn{4}{|l|}{ MEAN MEMORY SCORE } \\
\hline Word sequence learning-recall & $3.4(1.7)^{*}$ & $0.2(0.4)^{\#}$ & $0.0(0.0)^{\#}$ \\
\hline Visual reproduction II & $12.1(2.7)^{\star}$ & $8.0(2.9)^{\#}$ & $5.3(1.3)^{\# \$}$ \\
\hline Logic memory II & $12.3(2.8)^{\star}$ & $6.7(3.9)^{\#}$ & $2.4(1.1)^{\# \$}$ \\
\hline \multicolumn{4}{|c|}{ MEAN EXECUTIVE FUNCTION SCORE } \\
\hline $\begin{array}{l}\text { Semantic association of verbal } \\
\text { fluency }\end{array}$ & $34.8(7.1)^{\star}$ & $28.7(6.9)^{\#}$ & $15.4(7.4)^{\# \$}$ \\
\hline WCST-S completed categories & $5.4(1.1)^{*}$ & $3.4(1.9)^{\#}$ & $1.7(2.9)^{\#}$ \\
\hline WAIS-III digit symbol-scaled & $10.7(2.7)^{*}$ & $8.5(3.3)$ & $5.0(2.7)^{\# \$}$ \\
\hline WAIS-III digit span-scaled & $11.7(2.8)^{*}$ & $9.8(2.8)$ & $7.6(2.3)^{\#}$ \\
\hline \multicolumn{4}{|l|}{ MEAN VISUOSPATIAL SCORE } \\
\hline 3-D block construction models & $28.7(0.6)^{\star}$ & $26.9(3.7)$ & $19.1(10.1)^{\# \$}$ \\
\hline \multicolumn{4}{|l|}{ MEAN LANGUAGE SCORE } \\
\hline Object naming test & $16.0(0.0)^{\star}$ & $15.9(0.5)$ & $13.1(4.4)^{\# \$}$ \\
\hline
\end{tabular}

Mean (standard deviation): MMSE, Mini-mental state examination; WCST-S, Wisconsin Card Sorting Test-short form; WAIS-III, Wechsler Adult Intelligence Scale-III; *indicates significant difference among three groups. \#indicates significant difference between patients and normal controls. ${ }^{\$}$ indicates significant different between aMCl and $A D$ patients.

fluid-attenuated inversion recovery and $\mathrm{T}_{1}$-weighted magnetization-prepared rapid acquisition gradient echo (T1MPRAGE) images were acquired to rule out the concomitant neurological disorders. T1-MPRAGE images were subsequently used in post-processing for data normalization. The following imaging parameters were used: repetition time (TR)/echo time $(\mathrm{TE})=2000 \mathrm{~ms} / 2.63 \mathrm{~ms}, 160$ axial slices of voxel size $=1 \times 1$ $\times 1 \mathrm{~mm}^{3}$, inversion time $=900 \mathrm{~ms}$, flip angle $=9$ degree. The single acquisition time was $4 \mathrm{~min} 08 \mathrm{~s}$.

Diffusion weighted images were acquired using spin-echo echo planar imaging (EPI) sequence with the following parameters: TR/TE $=7324 \mathrm{~ms} / 83 \mathrm{~ms}, 64$ axial slices of voxel size $=2 \times 2 \times 2 \mathrm{~mm}^{3}, b$-value $1000 \mathrm{~s} / \mathrm{mm}^{2}$ with the diffusion weighted gradient applied along 64 non-collinear directions. The single average acquisition time was $8 \mathrm{~min} 47 \mathrm{~s}$.

\section{Image Processing and Analysis The Cortical Parcellation}

The procedure of image parcellation followed $\mathrm{Lu}$ et al. (2016) by homemade code in MATLAB (MATLAB 2013a. The MathWorks, Inc., Natick, Massachusetts, United States). 
The calculation and spatial normalization of the diffusion tensor were performed with Camino toolbox (Cook et al., 2006) (Microstructure Imaging Group-University College London) following the recommended procedure and parameters. The MD values were parcellated according to the standard automatic anatomic labeling (AAL) template following $\mathrm{Ng}$ et al. (2013). Briefly, non-diffusion weighted image (B0) from each individual was normalized to the ICBM 152 template from Montreal Neurological Institute (MNI) space 12-parameter affine transformation. The parameters used in the transformation were subsequently applied to MD of the same individual. The normalized MD was parcellated according to the AAL atlas. The mean values of MD from the 90 cortical regions were used in the final analysis.

\section{Track-Based Spatial Statistics}

In order to determine the extent of white matter involvement, track-based spatial statistics (TBSS, part of FMRIB Software Library Smith et al., 2004) was performed following its recommended procedure and settings. The difference in white matter skeleton between groups was calculated using Randomized permutation program (with 100,000 times permutations) (Nichols and Holmes, 2002).

\section{Voxel-Based Morphometry}

Brain atrophy was assessed by Voxel-Based Morphometry (VBM) using the analysis toolbox from Statistical Parametric Mapping 8 (Wellcome Department of Imaging Neuroscience, London (Friston et al., 1994). The T1-MPRAGE images from all subjects were normalized to the standard MNI space and segmented into gray matter, white matter as well as cerebrospinal fluid within the same generative model. The normalized gray matter images were smoothed with a Gaussian kernel $8 \times 8 \times$ $8 \mathrm{~mm}$ FWHM.

\section{Statistical Analyses}

All the statistical analyses were performed in PASW Statistics 21 (IBM Corp. Released 2012. IBM SPSS Statistics for Windows. Armonk, NY: IBM Corp.). The threshold of statistical significance was considered with Bonferroni post hoc correction. In the comparison of mean values among subject groups including the clinical neuropsychological scores and cortical $\mathrm{MD}$ values, one-way Analysis of variance (ANOVA) with post hoc multiple comparisons (Tukey honestly significant difference test). The statistical significance level was based on $p<0.05$. Correction for multiple comparison was applied by using Bonferroni method ( $p$-value $<0.0005$, Bonferroni: 0.05/90). The correlation between cortical MD and the neuropsychological scores was performed with controlled aging effect after Bonferroni multiple comparison correction ( $p$-value $<5.6 \mathrm{E}-5$, Bonferroni: 0.05/900).

In TBSS, family wise error (FWE) correction was performed to correct for multiple comparison, with a threshold of $p<$ 0.05 . The abnormal white matter tracts were identified based on the atlas prepared at Johns Hopkins University (Wakana et al., 2004). In VBM, differences in gray matter between groups were assessed using two-sample $t$-test with threshold of $p<0.05$ at false discovery rate (FDR).

\section{RESULTS}

\section{Clinical Evaluation}

Among the groups (NC, aMCI, and AD), no significant difference was noticed in gender, age and education level (Table 1). Compared to NC, AD patients had significant declination in all neuropsychological tests $(p<0.001)$. Compared to aMCI patients, $\mathrm{AD}$ patients showed reduced cognitive function in all tasks except for the learning-recall test of memory function, the completed categories test of executive function and the object naming test of language function. In addition, patients with aMCI had disturbed functions in the mean global and memory functions, semantic association of verbal fluency and the complete categories of Wisconsin card sorting test. No Apolipoprotein $\varepsilon 4$ (ApoE4) carriers were found in NC. Nine of 41 aMCI patients and 4 of $28 \mathrm{AD}$ patients were ApoE4 carrier.

\section{Gray Matter Changes in Patients}

Compared to NC, aMCI showed increased cortical MD in the right fusiform. In contrast, wide spread regions (Figure 1A, violet colored, 39 AAL regions) with significant increase MD can be found in $\mathrm{AD}$ patients, mainly located within the temporal lobe and cingulate gyrus. When comparing $\mathrm{AD}$ patients to aMCI, significantly increased $\mathrm{MD}$ was noticed in 10 cortical regions (Figure 1B, violet colored), including the bilateral hippocampus, bilateral posterior cingulum, right anterior cingulum, right amygdala, right caudate and left cuneus.

Both patients with aMCI and AD performed volumetric loss in cortical regions. The atrophy regions in aMCI were found in bilateral hippocampus and right parahippocampal gyrus, when compared to NC. In contrast, atrophy regions were found in $\mathrm{AD}$ patients mostly in temporal, parietal and frontal lobes, including the hippocampus, insula, fusiform, superior temporal gyrus, olfactory, and the inferior frontal triangularis (Figure 1A, warm colors). In addition, AD patients showed atrophic regions mainly hippocampus, fusiform gyrus, insula, olfactory gyrus, middle temporal gyrus and inferior frontal triangularis when compared to aMCI (Figure 1B, warm colors).

\section{Increased Cortical MD in AD and aMCI Patients}

The increased MD pattern among patients with aMCI and $\mathrm{AD}$ was showed in Figure 2. Initial $\mathrm{MD}$ increase can be observed in aMCI patients only located in the right fusiform (green color), when compared to NC. Compared to aMCI patients, $\mathrm{AD}$ patients showed expended regions of increased MD (yellow color), such as hippocampus, parahippocampus, amygdala, inferior temporal gyrus, cuneus, precuneus, anterior cingulum and posterior cingulum. In the later stage, increaed MD regions can be observed in every lobe of cortical brain in $\mathrm{AD}$ patinets (red color), compared to NC. Significant difference in the $\mathrm{MD}$ values were found in different cortical regions among 


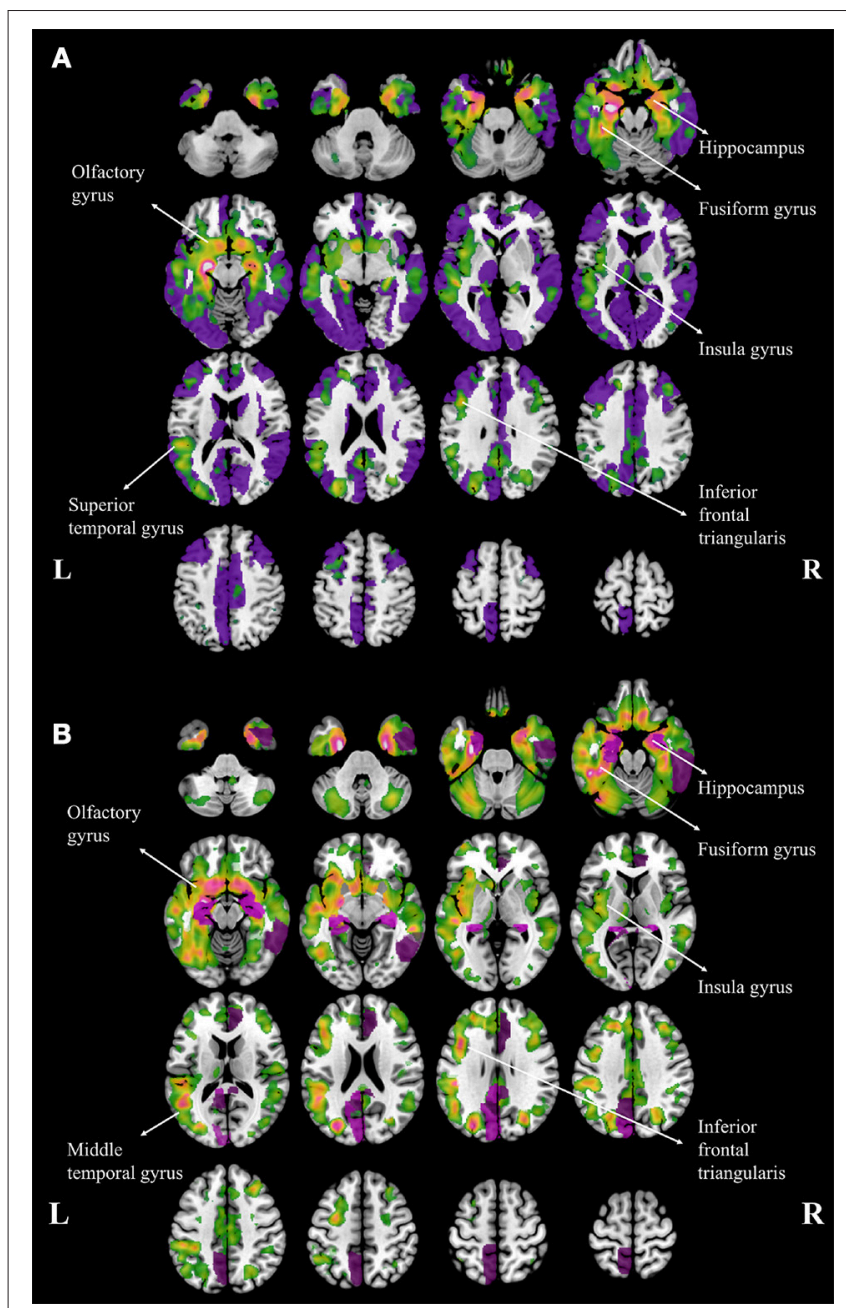

FIGURE 1 | Regions with increased mean diffusivity (MD) and gray matter loss in Alzheimer's disease (AD) patients, compared to normal controls $(A)$ and amenstic mild cognitive impairment $(\mathrm{aMCl})$ patients (B). The regions with significant increase MD are plotted with violet and the regions with gary matter atophy are plotted with warm colors, and the brighter color means the more severe atrophy in gray matter. The major atrophy regions were labeled with anatomy.

the groups in comparison (Figure 3, stars: between $\mathrm{AD}$ and NC; pounds: aMCI and NC; plus: aMCI and AD). Although both patient groups have significantly increased MD in cortical regions, the amount of change and the affected regions from the aMCI patients is always less than AD. The left hippocampus was the site with the most significant increase $(p<0.00001$, $12.51 \%$ increase in $\mathrm{AD}$ patients), when compared to NC. In the affected regions, $\mathrm{MD}$ increased by $7.52 \%$ in $\mathrm{AD}$ patients on average.

\section{White Matter Changes in Patients}

To identify the difference in the involvement of white matter, the difference of TBSS in between aMCI and NC was shown in Figure 4. Significant changes can be noticed in right superior longitudinal fasciculus (SLF), internal and external

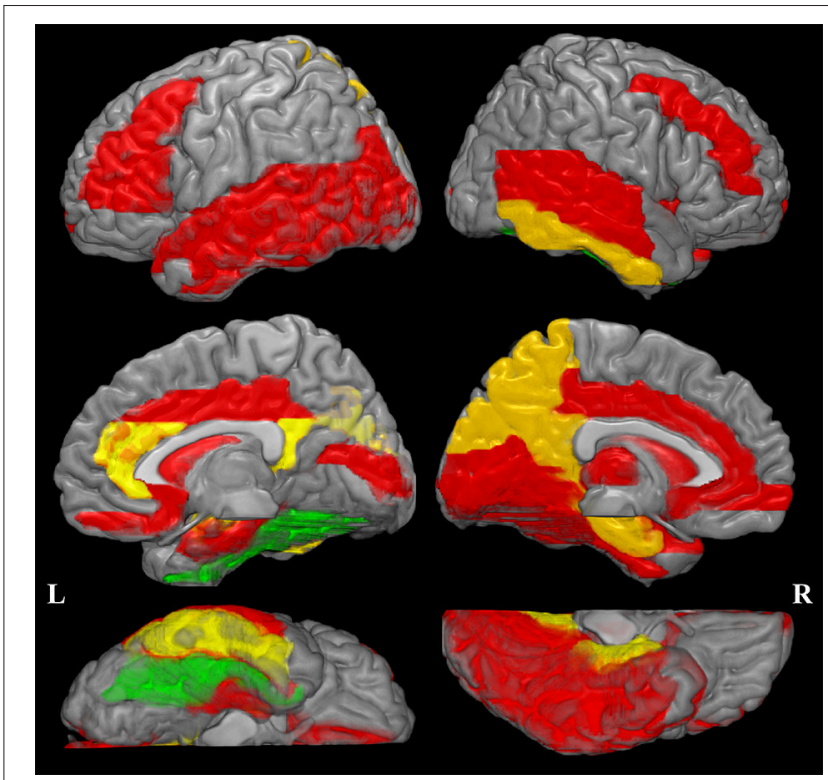

FIGURE 2 | Increased mean diffusivity (MD) pattern among patint with amenstic mild cognitive impairment (aMCl) and Alzheimer's disease (AD). Initial MD increase can be observed in aMCl patients only located in the right fusiform (violet color), when compared to normal controls (NC). Compared to $\mathrm{aMCl}$ patients, $\mathrm{AD}$ patients showed expended regions of increased $\mathrm{MD}$ (yellow color). In the later stage, increaed MD regions can be observed in every lobe of cortical brain in AD patinets (red color), compared to NC. This spatial distribution pattern of regions with increased $\mathrm{MD}$ is similar to the post-mortem pathology of amyloid deposition in AD patients (Braak and Braak, 1991).

capsule (Figure 4A). A general increase of $\mathrm{MD}$ was noticed throughout the whole brain (Figure 4B). However, no significant difference in white matter was found between the aMCI and AD patients.

\section{Correlation Analysis in aMCI and AD Patients}

Table 2 summarizes the cortical regions with significant correlation between $\mathrm{MD}$ and neuropsychological scores. Hippocampus and its neighborhood in the temporal lobe, such as fusiform gyrus and inferior temporal gyrus, are the main regions with significant correlation the functions of interest.

$\mathrm{MD}$ in the olfactory cortex and amygdala were significantly correlated with scores of Mini-Mental State Examination (MMSE) and Clinical Dementia Rating (CDR). The learning recall score of memory function was significantly correlated in regions located in the inferior frontal gyrus pars triangularis. The regions with correlation to semantic verbal fluency appeared to be scattered in the whole brain. The Wechsler Adult Intelligence Scale (WAIS-III) digit symbol-scaled score were significantly correlated with regions located in calcarine and lingual gyrus. In the visuospatial test, the 3-D block construction score significantly correlated with the cingulum, middle occipital, cuneus, lingual and angular gyrus. In the language function test, the object naming score was correlated with inferior parietal 


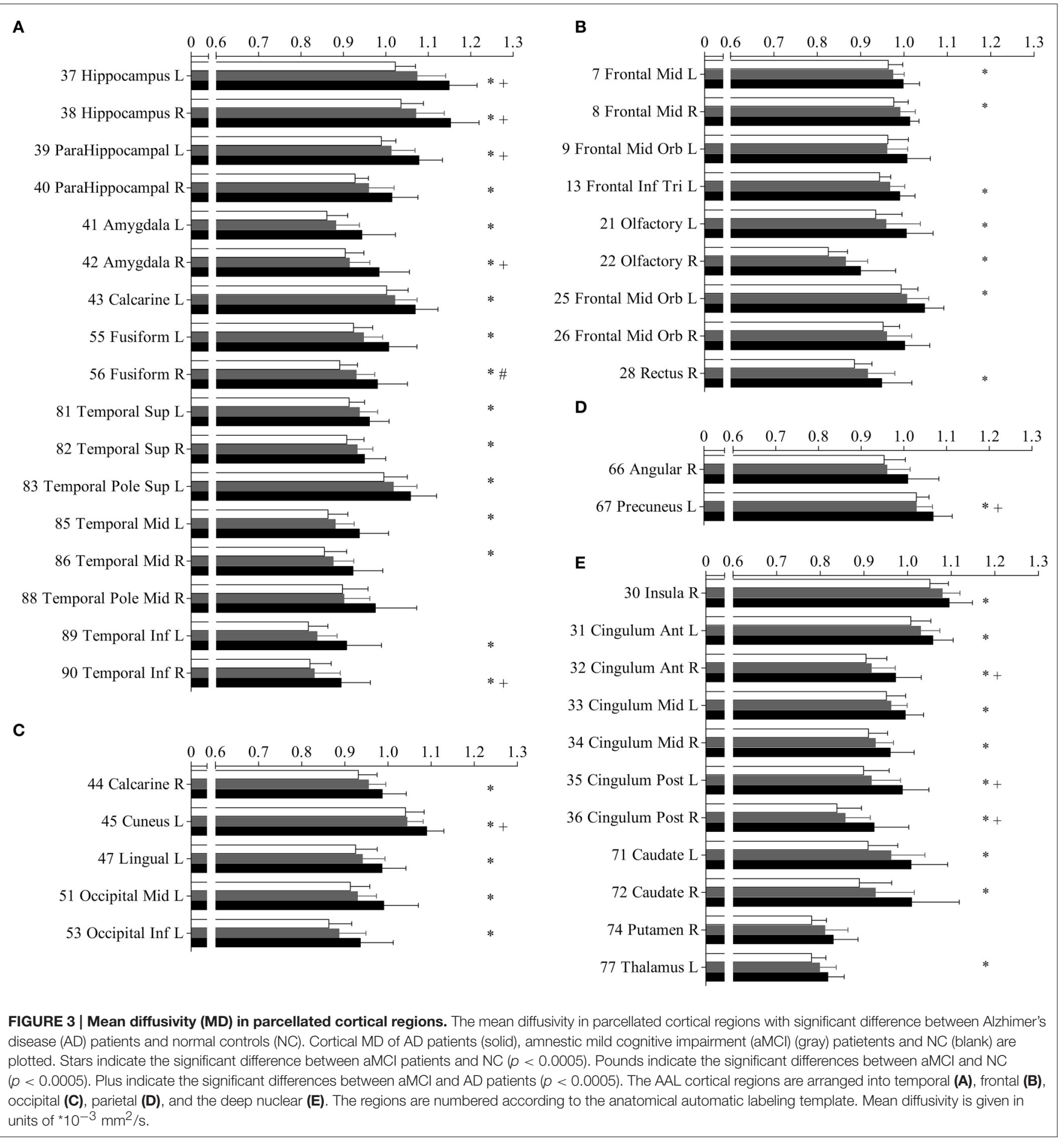

and angular gyrus. No significant correlation was found between education level and MD.

\section{DISCUSSION}

\section{Major Findings}

The current study reported an increased number of affected cortical regions and with growing difference, as the disease progressed from aMCI to AD. We measured the cortical mean diffusivity from two patient groups in comparison to the NC. Significant differences between $\mathrm{AD}$ and aMCI groups can be noticed in two aspects: the spatial pattern of the affected regions and the magnitude of increase. Although the atrophic pattern in $\mathrm{AD}$ suggested the whole brain involvement, the current study provided image based in vivo evidence that the microstructural damage might begin at an earlier stage. 


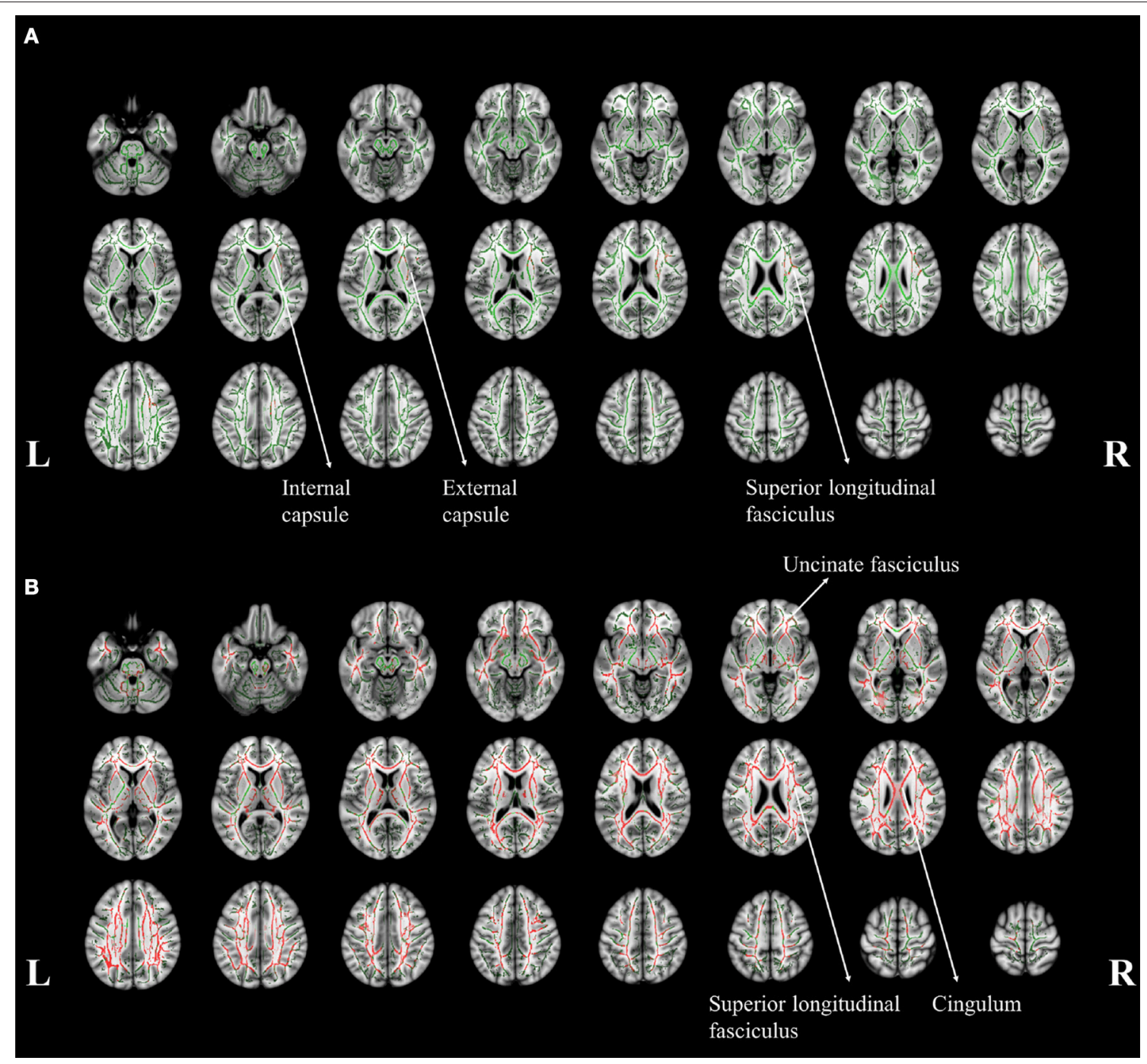

FIGURE 4 | The tract-based spatial statistics results of aMCI patients (A) and AD patients (B), when compared to normal controls. The white matter tract skelotons are plotted in green lines and the pixels with significant MD increase are marked with red on tracts. The major regions with increased MD were labeled with anatomy.

The spatial distribution suggested the involved regions might extend beyond the temporal lobe. The increase in mean diffusivity, a reflection of the severity in damage, indicated a widespread cortical impairment from MCI to AD. This spatial distribution pattern of regions with increased $\mathrm{MD}$ is similar to the post mortem pathology of amyloid deposition in $\mathrm{AD}$ patients (Braak and Braak, 1991). Furthermore, the affected region is consistent with that from the volumetric atrophy. Because the increase of $\mathrm{MD}$ is often attributed to an increase from the extracellular volume (Syková, 2004), as a result of the potential cell death, our observation might suggest a microstructural damage due to cellular degeneration or neural demyelination (Basser and Pierpaoli, 1996). Besides, significant correlations between the MD from the affected regions and the neuropsychological scores were observed.

\section{Increased MD in aMCI Patients}

In the aMCI patients, the current study observed significant atrophy located in the bilateral hippocampus, with increased $\mathrm{MD}$ in the peripheral region, noticeably fusiform gyrus. Only the right fusiform showed significant increase in $\mathrm{MD}(p=$ 0.0005 , percentage increase of $4.35 \%)$. Similar increase of MD was noticed in bilateral hippocampi (Left side: $p=0.001$, increase $=4.98 \%$; Right side: $p=0.03$, increase $=3.37 \%$ ). Although the difference did not reach statistical significance, the percentage increase from left hippocampus is even higher than that in the right fusiform.

The white matter damage indicated that a more distant region could be affected such as SLF (Figure 4A). Post-mortem study showed that the brain degeneration in aMCI (Braak and Braak, 1991) started from the hippocampus and spread into the surrounding area in the later stages. The increased cortical 
TABLE 2 | Regions with significant correlation between MD and neuropsychological scores.

Neuropsychological scores

MMSE
Cortical region (correlation coefficient, $p$-value)

Olfa. R. (-0.41, 1.21E-5)

Cing. Post. L. (-0.46, 8.67E-7)

Hipp. L. (-0.53, 6.90E-9)

ParaHipp. L. (-0.54, 1.23E-9)

Calc. L. (-0.43, 2.79E-6)

Ling L. (-0.43, 4.07E-6)

Fusi. L. (-0.58, 5.51E-11)

Caud. R. (-0.42, 9.08E-6)

Temp. Inf. L. (-0.45, 1.51E-6)

Olfa. R. (0.42, 1.03E-5)

Hipp.R. (0.54, 3.02E-9)

ParaHipp. R. (0.47, 6.07E-7)

Amyg. R. (0.49, 1.21E-7)

Fusi. R. (0.51, 3.59E-8)

Caud. R. (0.43, 3.76E-6)

\section{MEMORY FUNCTION}

Learning recall

Front. Inf. Oper. R. (-0.40, 4.06E-5)

Fusi. R. (-0.40, 3.64E-5)

Visual reprodiction

Hipp. L. (-0.46, 1.54E-6)

ParaHipp. L. (-0.53, 1.00E-8)

Amyg. L. (-0.40, 5.14E-5)

Fusi. R. (-0.50, 9.87E-8)

Hipp. L. (-0.56, 7.80E-10)

ParaHipp. L. (-0.52, 1.49E-8)

Amyg. L. (-0.54, 3.38E-6)

Fusi. L. (-0.50, 6.71E-8)

Temp. Inf. L. (-0.45, 1.87E-6)

\section{EXECUTIVE FUNCTION}

Semantic association of verbal fluency
Front. Sup. Orb. L. (-0.43, 5.25E-6)

Front. Mid. R. (-0.45, 1.63E-5)

Front. Inf. Oper. R. (-0.41, 2.05E-5)

Olfa. L. (-0.51, 4.25E-8)

Cing. Ant. L. (-0.48, 2.93E-7)

Cing. Ant. R. (-0.45, 1.92E-6)

Cing. Post R. (-0.43, 5.21E-6)

Hipp. R. (-0.51, 3.21E-8)

ParaHipp. R. (-0.59, 8.46E-11)

Calc. L. (-0.44, 3.83E-6)

Cuneus L. (-0.41, 1.61E-5)

Fusi. L. (-0.62, 2.77E-12)

Precuneus L. (-0.44, 3.33E-6)

Caud. R. (-0.45, 1.80E-6)

Temp. Sup. R. (-0.41, 1.73E-5)

Temp. Mid. R. (-0.47, 5.53E-7)

Temp. Pole Mid. R. (-0.41, 1.79E-5)
Cing. Ant. R. (-0.41, 9.62E-6)

Cing. Post. R. (-0.45, 1.22E-6)

Hipp. R. (-0.50, 4.83E-8)

ParaHipp. R. (-0.44, 3.77E-6)

Calc. R. (-0.38, 4.79E-5)

Occi. Mid. L. (-0.39, 4.13E-5)

Fusi. R. (-0.53, 5.26E-9)

Thal. L. (-0.38, 5.09E-5)

Hipp. L. (0.44, 2.00E-6)

ParaHipp. L. (0.44, 2.25E-6)

Amyg. L. (0.41, 2.72E-5)

Fusi. L. (0.44, 1.54E-6)

Caud. L. (0.42, 7.39E-6)

Temp. Inf. L. (0.40, 1.98E-5)

Hipp. L. (-0.48, 5.82E-7)

Hipp. R. (-0.41, 1.65E-5)

ParaHipp. R. (-0.50, 7.91E-8)

Fusi. L. (-0.52, 2.31E-8)

Temp. Inf. L. (-0.40, 3.12E-5)

Hipp. R. (-0.48, 3.64E-7)

ParaHipp. R. (-0.53, 1.11E-8)

Calc. L. (-0.44, 2.91E-5)

Fusi. R. (-0.50, 1.45E-7)

Front. Mid. L. (-0.40, 3.00E-5)

Front. Mid. Orb. L. (-0.43, 6.80E-6)

Front. Inf. Tri. L. (-0.41, 1.64E-5)

Olfa. R. (-0.45, 2.71E-6)

Cing. Post L. (-0.47, 5.75E-7)

Hipp. L. (-0.50, 9.70E-8)

ParaHipp. L. (-0.49, 1.67E-7)

Amyg. R. (-0.41, 2.53E-5)

Calc. R. (-0.46, 9.59E-7)

Ling. L. (-0.48, 3.16E-7)

Fusi. R. (-0.54, 7.71E-9)

Caud. L. (-0.45, 1.97E-6)

Temp. Sup. L. (-0.43, 6.01E-6)

Temp. Mid. L. (-0.57, 3.56E-10)

Temp. Pole Mid. L. (-0.42, 1.21E-5)

Temp. Inf. L. (-0.60, 2.42E-11)

Temp. Inf. R. (-0.46, 1.16E-6)
WCST-S completed categories

WAIS-III digit symbol-scaled

ParaHipp. R. (-0.43, 1.11E-5)

Hipp. L. $(-0.45,7.81 \mathrm{E}-6)$

Hipp. R. (-0.44, 1.49E-5)

(Continued) 


\begin{tabular}{|c|c|c|}
\hline \multirow[t]{3}{*}{ Neuropsychological scores } & \multicolumn{2}{|c|}{ Cortical region (correlation coefficient, $p$-value) } \\
\hline & ParaHipp. R. (-0.43, 2.64E-5) & Calc. L. (-0.42, 2.84E-5) \\
\hline & Ling. L. (-0.41, 4.11E-5) & Fusi. L. (-0.49, 7.46E-7) \\
\hline \multicolumn{3}{|l|}{ VISUALSPATIAL FUNCTION } \\
\hline \multirow[t]{6}{*}{ 3-D block construction Models } & Cing. Ant. R. (-0.39, 4.16E-5) & Cing. Mid. R. (-0.40, 3.05E-5) \\
\hline & Cuneus L. $(-0.49,1.39 \mathrm{E}-7)$ & Ling. L. (-0.41, 2.00E-5) \\
\hline & Occi. Mid. L. (-0.55, 2.56E-9) & Occi. Inf. L. $(-0.41,1.94 \mathrm{E}-5)$ \\
\hline & Fusi. L. (-0.54, 4.05E-9) & Fusi. R. (-0.48, 3.79E-7) \\
\hline & Angular L. (-0.39, 5.07E-5) & Angular R. (-0.43, 5.53E-6) \\
\hline & Precuneus L. (-0.43, 6.85E-6) & Temp. Mid. L. (-0.41, 1.71E-5) \\
\hline \multirow{8}{*}{ Object naming test } & ParaHipp. L. (-0.51, 2.39E-8) & Amyg. L. (-0.41, 3.19E-5) \\
\hline & Amyg. R. (-0.44, 4.28E-6) & Occi. Mid. L. (-0.49, 1.13E-7) \\
\hline & Fusi. L. (-0.59, 5.47E-11) & Fusi. R. (-0.54, 6.17E-9) \\
\hline & Parietal Sup. L. (-0.39, 3.46E-5) & Parietal Sup. R. (-0.39, 3.41E-5) \\
\hline & Parietal Inf. L. (-0.47, 5.88E-7) & Angular L. (-0.41, 1.51E-5) \\
\hline & Temp. Sup. L. (-0.39, 3.19E-5) & Temp. Pole Sup. L. (-0.47, 4.77E-7) \\
\hline & Temp. Mid. L. (-0.60, 1.68E-11) & Temp. Mid. R. (-0.43, 5.46E-6) \\
\hline & Temp. Pole Mid. R. (-0.46, 6.21E-7) & Temp. Inf. L. (-0.66, 2.74E-14) \\
\hline
\end{tabular}

Regions with significant correlation between to MD and neurophyscological scores. R., right side; L., Left side; Front. Sup. Orb., superior frontal gyrus; Front. Mid., Middle frontal gyrus; Front. Inf. Oper, opercular part of inferior frontal gyrus; Front. Inf. Tri., triangular part of inferior frontal gyrus; Olfa., olfactory gyrus; Cing. Ant., anterior cingulum; Cing. Mid., middle part of cingulum; Cing. Post, posterior cingulum; Hipp., hippocampus; ParaHipp., parahippocampal gyrus; Amyg., amygdala; Calc., calcarine gyrus; Cuneus, cuneus gyrus; Ling., lingual gyrus; Occi. Mid., middle occipital gyrus; Occi. Inf., inferior occipital gyrus; Fusi., fusiform gyrus; Parietal Sup., Superior parietal gyrus; Parietal Inf., inferior parietal gyrus; Angular, angular gyrus; Caud., caudate nucleus; Thal., thalamus; Temp. Sup., superior temporal gyrus; Temp. Pole Sup., temporal pole of superior temporal gyrus; Temp. Mid., middle temporal gyrus; Temp. Pole Mid., temporal pole of middle temporal gyrus; Temp. Inf., inferior temporal gyrus.

MD in right fusiform might be related to the functions of word recognition and within-category identification (McCarthy et al., 1997). The finding is consistent with the reduced performance in verbal fluency and completed categories tasks in this specific group of patients. The gray matter atrophy pattern in the hippocampus might be related to the potential damage in memory formation and storage (Burgess et al., 2002), as observed in the impaired memory functions.

Increased $\mathrm{MD}$ of the right SLF might suggest declination in the microstructural integrity within this region. The SLF structurally connects with the temporal, occipital and frontal lobes, which is functionally associated with the memory formation and higher cognitive functions such as verbal fluency (Kamali et al., 2014). In addition, the loss of integrity of white matter tracts which connected to gray matter with memory function has been associated to AD-risk (Gold et al., 2012). Increased MD in SLF could be potentially more sensitive to neurodegeneration than the volumetric atrophy.

\section{Affected Neural Network in AD Patients}

Increased $\mathrm{MD}$ was observed in widespread cortical regions of the $\mathrm{AD}$ patients. The spatial distribution of the involved regions was similar to that in beta-amyloid deposition and neurofibrillary tangle (Braak and Braak, 1991). This similarity might suggest possible association between changes in MD and the disruption within the neuronal environment. This spatial pattern of increased MD is also consistent with gray matter atrophy, similar to the previous report (Burton et al., 2009). The region with the most significant increase of $\mathrm{MD}$ and the most severe volumetric atrophy are both located in the hippocampus. Because hippocampus and the entorhinal cortex are involved in consolidating information from short-term memory into longterm memory (Schwindt and Black, 2009), the dysfunction in these regions are the hallmark of the AD pathology (Braak and Braak, 1991; Du et al., 2001). The increase of MD in hippocampus might attribute to the memory loss in our study (Table 1).

The affected white matter tracts in $\mathrm{AD}$ mainly located in bilateral uncinate fasciculus, SLF, and cingulum (Figure 4B). These affected tracts involves in the connection between the frontal, temporal and occipital lobes in neocortex (Friederici, 2011). It can be noticed that the cortical MD in the surrounding area was significantly increased. This observation might suggest that a more extensive neural network might be involved, as reflected in the increase of MD. In addition, the degeneration of the cingulum involved the development of cognitive impairment in AD (Bozzali et al., 2012), which might 
refer to the significant cognitive dysfunction in our $\mathrm{AD}$ patient group.

\section{Enhanced Cortical Involvement from aMCI to $A D$}

Compared with aMCI, more extensive cortical regions were involved in $\mathrm{AD}$ patients (one cortical region in aMCI patient and 40 in $\mathrm{AD}$ patients). Both the increase of $\mathrm{MD}$ and the involved regions suggest that the aMCI patients in the current study are in a transition state between the $\mathrm{NC}$ and $\mathrm{AD}$ patients. More severe brain degeneration in the more severe brain degeneration can be observed in $\mathrm{AD}$ patients when compared to aMCI. However, in term of white matter involvement, no significant difference between $\mathrm{AD}$ patients and aMCI can be found. This observation might support the fact that white matter changes could be secondary to the neurodegeneration in $\mathrm{AD}$ type dementia.

The current study noticed an overlap between the regions of gray matter atrophy and the more extensive and widespread areas of increased MD (Figure 1). The neurodegeneration process (Tripathi, 2012) begins with the loss of neural integrity, which subsequently evolve into massive cell death and shrinkage, and thus ultimately resulted in volumetric reduction and brain atrophy. The increase of MD is often attributed to the underlying microstructural damage. Because the spatial distribution pattern of the involved regions, as indicated by the increase of mean diffusivity, is similar to the post-mortem pathology of amyloid deposition in AD (Braak and Braak, 1991) and is more extensive than the atrophy map. It might suggest an increased sensitivity in the changes of MD than tissue atrophy. This observation certainly warrants new interest in further investigation in the future.

\section{Correlation between MD and Neuropsychological Performance}

Hippocampus, fusiform and inferior temporal gyrus were mainly negative correlated with the cognitive evaluation scores. The previous studies reported that the hippocampus atrophy associated with not only loss of memory formation but also executive dysfunction such as non-memory cognitive impairment in early $\mathrm{AD}$ and aMCI patients (Burgess et al., 2002; Nagata et al., 2011). The current finding suggested that the degeneration of hippocampus and its surroundings were not only the landmark of aMCI and $\mathrm{AD}$, but also involved the reduction of clinical cognitive performance in every aspect of evaluation including memory executive, visuospatial and language functions.

In addition to the hippocampus, different regions of cortical $\mathrm{MD}$ with significant correlation were noticed in different tasks for cognitive performance scores. Specifically, the memory function (the learning recall task) test showed negative correlation to MD in inferior frontal gyrus pars triangularis on left side, which has been known as a critical role in memory retrieval (Badre and Wagner, 2007). A scattered spatial pattern of cortical MD was also correlated with the semantic verbal fluency task of the executive function tests. The damage in cortical regions might interrupt the network of executive function including the ability of attention, planning and execution (Zhang et al., 2009; Nagata et al., 2011).

Deficits in visuospatial function in patients with $\mathrm{aMCI}$ and $\mathrm{AD}$ may suggest the loss of integrity of visual processing network. The middle occipital, cuneus and angular gyrus were noticed with significant correlation to the 3D block construction of the visuospatial test, which are critical roles involved the visuospatial information process (Bone, 2001). Interestingly, the regions with significant correlation between visuospatial function and cortical MD were mainly found on the left side. Although a lateralized brain network in visuospatial function were reported (Thiebaut de Schotten et al., 2011), the association between laterality of visuospatial function and cortical brain degeneration might need for further investigation.

\section{Effect of Apolipoprotein E $\varepsilon 4$ Allele}

Apolipoprotein $\varepsilon 4$ (ApoE4) carriers could be found in patients with $\mathrm{MCI}$ and $\mathrm{AD}$. The ApoE4 is the most widely known genetic risk factor for sporadic $\mathrm{AD}$ in different ethnic groups (Sadigh-Eteghad et al., 2012). However, Wai et al have reported a mild impact of the ApoE4 status on the extent of white matter disruption in the aMCI patients (Wai et al., 2014). The present study did not found significant trend between of the ApoE4 carrier and aMCI/AD. Number of ApoE4 carriers in aMCI/AD patient groups were smaller than non-carriers. Interestingly, the previous study has showed that the ApoE4 is less common in Chinese people than Caucasians or Japanese (Wong et al., 2005), which could explain the observation of leak of association between ApoE4 and AD/aMCI.

\section{Limitations}

The major limitation in the current study is a compromised spatial resolution in a template-based parcellation approach, each of which consisted of multiple voxels. This might lead to the potential statistical type I error. In a template-based parcellation approach, the MD value is an average from all voxels within the region of interest. If the observed effect within the region is heterogeneous, the statistical significance might be affected. Furthermore, the inhomogeneous nature and limited number of aMCI participants might reduce the sensitivity of detection. To establish a relationship between MD and the amyloid deposition is an interesting and important issue, which will have to be investigated in the animal study in the future because biopsy is impossible in human neurodegeneration study. Secondly, the diagnosis remains clinical, based on psychometric evaluation (Petersen, 2004). In the study design, only age and gender in the participants were matched. This data inhomogeneity, as shown in MMSE, is one major limitation in the current study, which to an extent reflected the difficulty in the diagnosis of the neurodegenerative disease, specifically Alzheimer Disease. Objective evidences such as neuroimaging-based and genomebased might improve the accuracy of clinical judgment. Finally, because the APOE status remained uncertain for a significant amount of the participants because they did not provide the blood sample for examination, this might lead to concerns in the reliability of the result for the effects of APOE in neuroimaging. 


\section{CONCLUSIONS}

The current study identified a specific spatial pattern in the cortex of increased $\mathrm{MD}$ from patients with $\mathrm{aMCI}$ or $\mathrm{AD}$. A correlation between MD and neuropsychological evaluations were reported between the functionally related cortical regions. The findings demonstrated the possibility of MD as an indicator at the early stage of cortical degeneration.

\section{AUTHOR CONTRIBUTIONS}

SL, WH, CH, and JW contributed to the design of the study, acquired the data and drafted a significant portion of the manuscript. SN, YLC, and YCC supervised the data acquisition. SL, JC, and OK analyzed the data and drafted a significant portion of the manuscript and figures. JW and SL initiated the concept and the design of the study, acquired and analyzed the data and completed the manuscript.

\section{REFERENCES}

Ahmed, S., Mitchell, J., Arnold, R., Nestor, P. J., and Hodges, J. R. (2008). Predicting rapid clinical progression in amnestic mild cognitive impairment. Dement. Geriatr. Cogn. Disord. 25, 170-177. doi: 10.1159/000113014

Alzheimer's Association (2014). Alzheimer's disease facts and figures. Alzheimers Dement. 10, e47-e92. doi: 10.1016/j.jalz.2014.02.001

Badre, D., and Wagner, A. D. (2007). Left ventrolateral prefrontal cortex and the cognitive control of memory. Neuropsychologia 45, 2883-2901. doi: 10.1016/j.neuropsychologia.2007.06.015

Basser, P. J., and Pierpaoli, C. (1996). Microstructural and physiological features of tissues elucidated by quantitative-diffusion-tensor MRI. J. Magn. Reson. B 111, 209-219. doi: 10.1006/jmrb.1996.0086

Bone, I. (2001). Medical neurosciences. An approach to anatomy, pathology and physiology by systems and levels. Brain 124, 447-448. doi: 10.1093/brain/124.2.447

Bozzali, M., Giulietti, G., Basile, B., Serra, L., Spanò, B., Perri, R., et al. (2012). Damage to the cingulum contributes to Alzheimer's disease pathophysiology by deafferentation mechanism. Hum. Brain Mapp. 33, 1295-1308. doi: 10.1002/hbm.21287

Braak, H., and Braak, E. (1991). Neuropathological stageing of Alzheimer-related changes. Acta Neuropathol. 82, 239-259. doi: 10.1007/BF00308809

Burgess, N., Maguire, E. A., and O'Keefe, J. (2002). The human hippocampus and spatial and episodic memory. Neuron 35, 625-641. doi: 10.1016/ S0896-6273(02)00830-9

Burton, E. J., Barber, R., Mukaetova-Ladinska, E. B., Robson, J., Perry, R. H., Jaros, E., et al. (2009). Medial temporal lobe atrophy on MRI differentiates Alzheimer's disease from dementia with Lewy bodies and vascular cognitive impairment: a prospective study with pathological verification of diagnosis. Brain 132(Pt 1), 195-203. doi: 10.1093/brain/awn298

Cook, P. A., Bai, Y., Nedjati-Gilani, S., Seunarine, K. K., Hall, M. G., Parker, G. J., et al. (2006). "Camino: open-source diffusion-MRI reconstruction and processing," in 14th Scientific Meeting of the International Society for Magnetic Resonance in Medicine (Seattle, WA), 2759.

Du, A. T., Schuff, N., Amend, D., Laakso, M. P., Hsu, Y. Y., Jagust, W. J., et al. (2001). Magnetic resonance imaging of the entorhinal cortex and hippocampus in mild cognitive impairment and Alzheimer's disease. J. Neurol. Neurosurg. Psychiatr. 71, 441-447. doi: 10.1136/jnnp.71.4.441

Duong, T. Q., Ackerman, J. J., Ying, H. S., and Neil, J. J. (1998). Evaluation of extraand intracellular apparent diffusion in normal and globally ischemic rat brain via 19F NMR. Magn. Reson. Med. 40, 1-13. doi: 10.1002/mrm. 1910400102

Fellgiebel, A., Wille, P., Müller, M. J., Winterer, G., Scheurich, A., Vucurevic, G., et al. (2004). Ultrastructural hippocampal and white matter alterations in mild

\section{FUNDING}

This work was supported by grants from the Ministry of Science and Technology Taiwan (MOST103-2325-B-182-001), Ministry of Education Taiwan (EMRPD1E1731) and the Chang-Gung Memorial Hospital (CMRPD1C0291-3 and CMRPD3D0011).

\section{ACKNOWLEDGMENTS}

The imaging facility was supported by the Imaging Core Laboratory of Institute for Radiological Research and Center for Advanced Molecular Imaging and Translation. The authors would like to thank the Neuroscience Research Center, Chang Gung Memorial Hospital and the Healthy Aging Center, Chang Gung University for providing support. The funding source had no involvement in the collection, analysis and interpretation data; in the writing of the report; and in the decision to submit the paper for publication.

cognitive impairment: a diffusion tensor imaging study. Dement. Geriatr. Cogn. Disord. 18, 101-108. doi: 10.1159/000077817

Friederici, A. D. (2011). The brain basis of language processing: from structure to function. Physiol. Rev. 91, 1357-1392. doi: 10.1152/physrev.000 06.2011

Friston, K. J., Jezzard, P., and Turner, R. (1994). Analysis of functional MRI time-series. Hum. Brain Mapp. 1, 153-171. doi: 10.1002/hbm.460010207

Gold, B. T., Johnson, N. F., Powell, D. K., and Smith, C. D. (2012). White matter integrity and vulnerability to Alzheimer's disease: preliminary findings and future directions. Biochim. Biophys. Acta 1822, 416-422. doi: 10.1016/j.bbadis.2011.07.009

Helenius, J., Soinne, L., Perkiö, J., Salonen, O., Kangasmäki, A., Kaste, M., et al. (2002). Diffusion-weighted MR imaging in normal human brains in various age groups. AJNR Am. J. Neuroradiol. 23, 194-199. Available online at: http:// www.ajnr.org/content/23/2/194.full

Kamali, A., Flanders, A. E., Brody, J., Hunter, J. V., and Hasan, K. M. (2014). Tracing superior longitudinal fasciculus connectivity in the human brain using high resolution diffusion tensor tractography. Brain Struct. Funct. 219, 269-281. doi: 10.1007/s00429-012-0498-y

Liu, Y., Spulber, G., Lehtimäki, K. K., Könönen, M., Hallikainen, I., Gröhn, H., et al. (2011). Diffusion tensor imaging and tract-based spatial statistics in Alzheimer's disease and mild cognitive impairment. Neurobiol. Aging 32, 1558-1571. doi: 10.1016/j.neurobiolaging.2009.10.006

Lu, C. S., Ng, S. H., Weng, Y. H., Cheng, J. S., Lin, W. Y., Wai, Y. Y., et al. (2016). Alterations of diffusion tensor MRI parameters in the brains of patients with Parkinson's disease compared with normal brains: possible diagnostic use. Eur. Radiol. 26, 3978-3988. doi: 10.1007/s00330-016-4232-7

McCarthy, G., Puce, A., Gore, J. C., and Allison, T. (1997). Face-specific processing in the human fusiform gyrus. J. Cogn. Neurosci. 9, 605-610. doi: $10.1162 /$ jocn.1997.9.5.605

McKhann, G., Drachman, D., Folstein, M., Katzman, R., Price, D., and Stadlan, E. M. (1984). Clinical diagnosis of Alzheimer's disease: report of the NINCDSADRDA Work group under the auspices of department of health and human services task force on Alzheimer's disease. Neurology 34, 939-944. doi: 10.1212/WNL.34.7.939

Mielke, M. M., Kozauer, N. A., Chan, K. C., George, M., Toroney, J., Zerrate, M., et al. (2009). Regionally-specific diffusion tensor imaging in mild cognitive impairment and Alzheimer's disease. Neuroimage 46, 47-55. doi: 10.1016/j.neuroimage.2009.01.054

Nagata, T., Shinagawa, S., Ochiai, Y., Aoki, R., Kasahara, H., Nukariya, K., et al. (2011). Association between executive dysfunction and hippocampal volume in Alzheimer's disease. Int. Psychogeriatr. 23, 764-771. doi: 10.1017/ S1041610210002164 
Ng, S. H., Hsu, W. C., Wai, Y. Y., Lee, J. D., Chan, H. L., Chen, Y. L., et al. (2013). Sex dimorphism of cortical water diffusion in normal aging measured by magnetic resonance imaging. Front. Aging Neurosci. 5:71. doi: $10.3389 /$ fnagi.2013.00071

Nichols, T. E., and Holmes, A. P. (2002). Nonparametric permutation tests for functional neuroimaging: a primer with examples. Hum. Brain Mapp. 15, 1-25. doi: $10.1002 / \mathrm{hbm} .1058$

Petersen, R. C. (2004). Mild cognitive impairment as a diagnostic entity. J. Intern. Med. 256, 183-194. doi: 10.1111/j.1365-2796.2004.01388.x

Petersen, R. C., Doody, R., Kurz, A., Mohs, R. C., Morris, J. C., Rabins, P. V., et al. (2001). Current concepts in mild cognitive impairment. Arch. Neurol. 58, 1985-1992. doi: 10.1001/archneur.58.12.1985

Petersen, R. C., Smith, G. E., Waring, S. C., Ivnik, R. J., Tangalos, E. G., and Kokmen, E. (1999). Mild cognitive impairment: clinical characterization and outcome. Arch. Neurol. 56, 303-308. doi: 10.1001/archneur.56.3.303

Prince, M., Albanese, E., Guerchet, M., and Prina, M. (2014). The World Alzheimer Report 2014, Dementia and Risk Reduction: An Analysis of Protective and Modifiable Factors. Alzheimer's Disease International.

Sadigh-Eteghad, S., Talebi, M., and Farhoudi, M. (2012). Association of apolipoprotein E epsilon 4 allele with sporadic late onset Alzheimer's disease. A meta-analysis. Neuroscience 17, 321-326. Available online at: https://pdfs. semanticscholar.org/ee03/5f306elac554c87400a8ec932979ad419c78.pdf

Schaefer, P. W., Grant, P. E., and Gonzalez, R. G. (2000). Diffusion-weighted MR imaging of the brain. Radiology 217, 331-345. doi: 10.1148/radiology. 217.2.r00nv24331

Schwindt, G. C., and Black, S. E. (2009). Functional imaging studies of episodic memory in Alzheimer's disease: a quantitative meta-analysis. Neuroimage 45, 181-190. doi: 10.1016/j.neuroimage.2008.11.024

Smith, S. M., Jenkinson, M., Woolrich, M. W., Beckmann, C. F., Behrens, T. E., Johansen-Berg, H., et al. (2004). Advances in functional and structural MR image analysis and implementation as FSL. Neuroimage 23(Suppl. 1), S208-S219. doi: 10.1016/j.neuroimage.2004.07.051

Syková, E. (2004). Diffusion properties of the brain in health and disease. Neurochem. Int. 45, 453-466. doi: 10.1016/j.neuint.2003.11.009

Thiebaut de Schotten, M., Dell'Acqua, F., Forkel, S. J., Simmons, A., Vergani, F., Murphy, D. G., et al. (2011). A lateralized brain network for visuospatial attention. Nat. Neurosci. 14, 1245-1246. doi: 10.1038/nn.2905
Thompson, P. M., Hayashi, K. M., Dutton, R. A., Chiang, M. C., Leow, A. D., Sowell, E. R., et al. (2007). Tracking Alzheimer's disease. Ann. N. Y. Acad. Sci. 1097, 183-214. doi: 10.1196/annals.13 79.017

Tripathi, A. (2012). New cellular and molecular approaches to ageing brain. Ann. Neurosci. 19, 177-182. doi: 10.5214/ans.0972.7531. 190410

Wai, Y. Y., Hsu, W. C., Fung, H. C., Lee, J. D., Chan, H. L., Tsai, M. L., et al. (2014). Tract-based spatial statistics: application to mild cognitive impairment. Biomed Res. Int. 2014:713079. doi: 10.1155/2014/713079

Wakana, S., Jiang, H., Nagae-Poetscher, L. M., van Zijl, P. C., and Mori, S. (2004). Fiber tract-based atlas of human white matter anatomy. Radiology 230, 77-87. doi: 10.1148/radiol.2301021640

Whitwell, J. L., Shiung, M. M., Przybelski, S. A., Weigand, S. D., Knopman, D. S., Boeve, B. F., et al. (2008). MRI patterns of atrophy associated with progression to $\mathrm{AD}$ in amnestic mild cognitive impairment. Neurology 70 , 512-520. doi: 10.1212/01.wnl.0000280575.77437.a2

Wong, S. Y., Lau, E. M., Li, M., Chung, T., Sham, A., and Woo, J. (2005). The prevalence of Apo E4 genotype and its relationship to bone mineral density in Hong Kong Chinese. J. Bone Miner. Metab. 23, 261-265. doi: 10.1007/s00774-004-0593-0

Zhang, Y., Schuff, N., Du, A. T., Rosen, H. J., Kramer, J. H., Gorno-Tempini, M. L., et al. (2009). White matter damage in frontotemporal dementia and Alzheimer's disease measured by diffusion MRI. Brain 132(Pt 9), 2579-2592. doi: 10.1093/brain/awp071

Conflict of Interest Statement: The authors declare that the research was conducted in the absence of any commercial or financial relationships that could be construed as a potential conflict of interest.

Copyright (c) 2017 Lin, Hsu, Ng, Cheng, Khegai, Huang, Chen, Chen and Wang. This is an open-access article distributed under the terms of the Creative Commons Attribution License (CC BY). The use, distribution or reproduction in other forums is permitted, provided the original author(s) or licensor are credited and that the original publication in this journal is cited, in accordance with accepted academic practice. No use, distribution or reproduction is permitted which does not comply with these terms. 